\title{
Urinary Excretion of microRNAs in Young Fabry Disease Patients with Mild or Absent Nephropathy
}

\section{Sebastián Jaurretche1,2*, Graciela Venera ${ }^{1}$, Norberto Antongiovanni ${ }^{3}$, Fernando Perretta ${ }^{4}$, Germán R. Perez 5,6}

\author{
${ }^{1}$ School of Medicine, Instituto Universitario Italiano de Rosario, Rosario, Argentina \\ ${ }^{2}$ Los Manantiales, Neurosciences Center, Rosario, Argentina. \\ ${ }^{3}$ Center for Infusion and Study of Lysosomal Diseases, Instituto de Nefrología de Pergamino, Pergamino, Argentina \\ ${ }^{4}$ Intensive Care Unit, Hospital Dr. Enrique Erill, Belen de Escobar, Argentina \\ ${ }^{5}$ School of Biochemical and Pharmaceutical Sciences, Nacional University of Rosario, Rosario, Argentina \\ ${ }^{6}$ Gammalab Grupo Gamma, Rosario, Argentina \\ Email: *sebastianjaurretche@hotmail.com
}

How to cite this paper: Jaurretche, S., Venera, G., Antongiovanni, N., Perretta, F. and Perez, G.R. (2018) Urinary Excretion of microRNAs in Young Fabry Disease Patients with Mild or Absent Nephropathy. Open Journal of Nephrology, 8, 71-83. https://doi.org/10.4236/ojneph.2018.83009

Received: August 23, 2018

Accepted: September 8, 2018

Published: September 11, 2018

Copyright (C) 2018 by authors and Scientific Research Publishing Inc. This work is licensed under the Creative Commons Attribution International License (CC BY 4.0).

http://creativecommons.org/licenses/by/4.0/

\section{Open Access}

\begin{abstract}
Fabry disease (FD) clinical manifestations often start in childhood. Among the FD complications, renal failure causes significant morbidity and mortality. Early diagnosis and treatment of FD nephropathy in children may be critical to preserve renal function. In proteinuric progressive nephropathies it has been described that pro-fibrotic miR-21, miR-192, and miR-433 families are activated and that anti-fibrotic miR-29 and miR-200 families are inhibited. Objective: Analyze urinary excretion of microRNAs related to renal fibrosis in FD patients with mild or absent nephropathy. Patients with confirmed diagnosis of FD under 18 years of age were compared with healthy subjects. Patients were classified into two groups: 1) Patients with urinary excretion profile of microRNAs indicative of renal fibrosis; and 2) Patients with urinary excretion profile of microRNAs not indicative of renal fibrosis. Results: 9 healthy subjects were enrolled in the study (18.66 \pm 13.43 years), 4 males and 5 females. All of them presented normal eFGR without pathological albuminuria. FD population: 12 patients $(10.33 \pm 3.93$ years $)$ were studied, 5 males and 7 females. Patients presented 2 different genotypes: L415P (6 patients) and E398X (6 patients). The urinary excretion profile of microRNAs indicative of renal fibrosis was present in 4 patients (2 with L415P genotype and 2 with E398X genotype), all of them with a decreased of miR-29 and/or miR-200. No patient presented increased miR-21, miR-192 and/or miR-433. Decreased $\alpha$-gal-A activity was the only variable associated with statistical significance $(\mathrm{p} \leq 0.01)$ to urinary excretion profile of microRNA indicative of
\end{abstract}


renal fibrosis. Conclusions: Young FD patients with classical mutations of GLA gene and mild or absent nephropathy could present a profile of urinary excretion of microRNAs indicative of renal fibrosis associated with decreased $\alpha$-gal-A activity independently of the other variables. Our findings could suggest a regulation of microRNAs not mediated by TGF- $\beta$ in FD nephropathy.

\section{Keywords}

Fabry Nephropathy, Biomarkers, Urinary microRNA, Renal Fibrosis, Early Diagnosis

\section{Introduction}

Fabry disease (FD) is a multi-organ disease, X-linked $\alpha$-galactosidase A ( $\alpha$-Gal-A) deficiency which results in the failure in the glycosphingolipids catabolism, particularly, and globotriaosylceramide (Gb3) [1]. The abnormal storage of Gb3 in the lysosomes and other cell compartments starts in fetal life [2], and leads to deteriorated cellular function which in turn causes the symptoms of the disease [1].

FD clinical manifestations often start in childhood and are seen in both males and females [1] [3]. Among the FD complications, renal failure causes significant morbidity and mortality [4]. Whereas in the majority of affected patients, renal involvement is clinically silent in childhood, once becoming evident as overt proteinuria, FD nephropathy leads progressively to renal failure [5].

Thus, measurements of proteinuria and estimated glomerular filtration rate (eGFR) with formulas that use serum creatinine are the cornerstones in the assessment and monitoring of kidney function [5]. However, these tests have low sensitivity in detecting early kidney damage and abnormal values are often late signs of kidney disease, already in the setting of at least partially irreversible structural damage [5]. Proteinuria, which is long considered the first clinical manifestation of FD nephropathy, may start as early as at 10 years of age [6]. It is well known that morphological changes, even irreversible, such as interstitial tubular fibrosis or glomerular sclerosis, precede the onset of pathological albuminuria [7] [8] [9].

Patients may not fully benefit from enzyme replacement therapy (ERT) if starting when proteinuria is clinically manifest or glomerular filtration rate has already declined. Thus, early diagnosis and treatment of FD nephropathy in children may be critical to preserve renal function [5].

Early biomarkers of Fabry disease nephropathy have been studied in affected adult patients with some promising results [10] [11] [12]. Several studies suggest the potential role of microRNAs in kidney function [13]. In others proteinuric progressive nephropathies it has been described that miR-21, miR-192, and miR-433 families are activated (pro-fibrotics), and that miR-29 and miR-200 families are inhibits (anti-fibrotics) [14]. 
In FD patients the expression of different circulating microRNAs related to cardiovascular complications has been studied [15] [16] [17] [18]. To date, no study has been published on the study of microRNAs related to renal fibrosis in FD young patients with mild or absent nephropathy.

The early indication of ERT in children and adolescents is controversial. It is recommended ERT start in the presence of clinical manifestations in affected patients of both sexes. In asymptomatic patients the time of ERT start is not so clear. While it is recommended to start arbitrarily at 8 to 10 years of age in children with "classic" mutations of the GLA gene; in asymptomatic girls and children with "late onset" mutations, the time of onset remains unclear [19].

The knowledge of early biomarkers of damage could help to better define the appropriate time for ERT start in FD patients at early ages. The purpose of this study is to analyze urinary excretion of microRNAs related to renal fibrosis in FD patients with mild or absent nephropathy.

\section{Material and Methods}

\subsection{Population}

Patients with confirmed diagnosis of FD by genetic test of any gender, under 18 years of age, were included. Exclusion criteria were taken for: 1) patients with nephropathy by different etiology to FD, 2) patients who at the time of evaluation had any symptoms or intercurrence, and 3) patients with confirmed diagnosis of FD who refused to participate in the study. The elimination criterion was applied to: patients who meet inclusion criteria and presented some complication related to the collection process of the samples. A population of healthy subjects with similar demographic characteristics was included.

The patients selected were fasting to the laboratory for the extraction of blood samples and first urine in the morning.

All patients had a mutational study by direct sequencing and Multiplex Ligations Probe Amplification (MLPA) [20] [21], and quantification of $\alpha$-gal-A enzymatic activity by fluorometric method [22]. Decreased or normal enzyme activity was considered at values less than or greater than $4.0 \mathrm{nmol} / \mathrm{h} / \mathrm{l}$, respectively. Plasma and urine creatinine were determined by electrochemiluminescence (Roche Diagnostics). Albuminuria was determined by colorimetric method (Roche Diagnostics). The urinary albumin/creatinine ratio was calculated to estimate 24 hour albuminuria [23]. Ratio values 0 to 30 were considered normal, microalbuminuria when 30 to 300 , and macroalbuminuria when higher than 300, in at least two samples. eGFR was calculated using Schwartz 09 (modification 2009) equation [24].

\subsection{Urine Sample Preparation and microRNAs Extraction}

Urine specimen was collected and sent to laboratory for processing immediately. A volume of $10 \mathrm{ml}$ of urine sample was centrifuged at $3000 \times \mathrm{g}$ for 15 minutes. $9 \mathrm{ml}$ of supernatant was discarded and the remaining milliliter was centrifuged at 
15000× g during 5 minutes at room temperature. The urinary cell pellet was stored at $-80^{\circ} \mathrm{C}$ until use. The extraction of microRNAs was performed according to the manufacturer's protocol (NucleoSpinmiRNA Plasma kits, Macheney-Nagel, Germany).

\subsection{Detection of microRNAs}

To detect the urinary expression of miR-21, miR-29 family, miR-192, miR-200 families and miR-433 in the urine sample, reverse transcription (RT) reaction with a stem-loop primer were used. Stem-loop RT primers were designed according to Chen et al. [25]. Sequence data are presented in Table 1. The specificity of the stem-loop RT primers of each microRNAs is determined by an extension of six nucleotides at the $3^{\prime}$ end; this extension is inverse and complementary to the last six nucleotides of the 3 ' end of the microRNA.

microRNAs were reverse transcribed using Transcriptor First Strand cDNA Synthesis Kit (Roche Diagnostics). Briefly, $5 \mu$ l total eluate was mixed with $1 \mu \mathrm{M}$ stem-loop RT primer, $0.5 \mu \mathrm{M}$ dNTPs, $1 \times$ RT reaction buffer, $20 \mathrm{U}$ RNase inhibitor,

Table 1. microRNA and primer sequences.

\begin{tabular}{|c|c|c|c|}
\hline \multirow{2}{*}{ microRNA } & \multicolumn{3}{|r|}{ Primers } \\
\hline & Type & name & Sequence \\
\hline \multirow[t]{2}{*}{$\operatorname{miR}-21$} & stem-loop primer & Loop. 21 & GTCGTATCCAGTGCAGGGTCCGAGGTATTCGCACTGGATACGACTCAACA \\
\hline & foward primer* & F. 21 & CACGCATAGCTTATCAGACTG \\
\hline \multirow[t]{3}{*}{$\operatorname{mirR}-29 \mathrm{a} / \mathrm{c}$} & stem-loop primer & Loop.29a & GTCGTATCCAGTGCAGGGTCCGAGGTATTCGCACTGGATACGACTAACCG \\
\hline & stem-loop primer & Loop.29c & GTCGTATCCAGTGCAGGGTCCGAGGTATTCGCACTGGATACGACTAACCG \\
\hline & foward primer ${ }^{*}$ & F.29a/c & CACGCATAGCACCATCTGAAA \\
\hline \multirow[t]{2}{*}{$\operatorname{mirR}-29 b$} & stem-loop primer & Loop.29b & GTCGTATCCAGTGCAGGGTCCGAGGTATTCGCACTGGATACGACTTCACT \\
\hline & foward primer* & F.29b & CACGCATAGCACCATTTGAAA \\
\hline \multirow[t]{2}{*}{ mirR-192 } & stem-loop primer & Loop.192 & GTCGTATCCAGTGCAGGGTCCGAGGTATTCGCACTGGATACGACGGCTGT \\
\hline & foward primer ${ }^{*}$ & F.192 & CACGCACTGACCTATGAATTG \\
\hline \multirow[t]{2}{*}{ mirR-200a } & stem-loop primer & Loop.200a & GTCGTATCCAGTGCAGGGTCCGAGGTATTCGCACTGGATACGACACATCG \\
\hline & foward primer ${ }^{*}$ & F.200a & CACGCATAACACTGTCTGGTA \\
\hline \multirow[t]{2}{*}{ mirR-200b } & stem-loop primer & Loop.200b & GTCGTATCCAGTGCAGGGTCCGAGGTATTCGCACTGGATACGACTCATCA \\
\hline & foward primer* & F.200b & CACGCATAATACTGCCTGGTA \\
\hline \multirow[t]{2}{*}{$\operatorname{mirR}-200 c$} & stem-loop primer & Loop.200c & GTCGTATCCAGTGCAGGGTCCGAGGTATTCGCACTGGATACGACTCCATC \\
\hline & foward primer* & F.200c & CACGCATAATACTGCCGGGTA \\
\hline \multirow[t]{2}{*}{$\operatorname{mirR}-433$} & stem-loop primer & Loop.433 & GTCGTATCCAGTGCAGGGTCCGAGGTATTCGCACTGGATACGACACACCG \\
\hline & foward primer & F.433 & CACGCAATCATGATGGGCTCC \\
\hline \multirow[t]{2}{*}{ mirR-U6 } & stem-loop primer & Loop.U6 & GTCGTATCCAGTGCAGGGTCCGAGGTATTCGCACTGGATACGACAAAATATGGAAC \\
\hline & foward primer ${ }^{*}$ & F.U6 & GCCCCTGCGCAAGGATGAC \\
\hline Universal & reverse primer ${ }^{\star}$ & R.MIR & CCAGTGCAGGGTCCGAGGTA \\
\hline
\end{tabular}

${ }^{\star}$ Hybridization temperature for qPCR: $60^{\circ} \mathrm{C}$. 
$10 \mathrm{U}$ Transcriptor RT and made up to $20 \mu \mathrm{l}$ with $\mathrm{H}_{2} \mathrm{O}$. RT was performed at $16^{\circ} \mathrm{C}$ for 30 minutes, $42^{\circ} \mathrm{C}$ for 30 minutes, $60^{\circ} \mathrm{C}$ for 60 minutes and $70^{\circ} \mathrm{C}$ for 15 minutes. The resulting $\mathrm{CDNA}$ was stored at $-80^{\circ} \mathrm{C}$ until use.

FastStart Universal SYBR Green Master/ROX (Roche Diagnostics) was used for the qPCR reaction, which was performed according manufacturer's protocol on a StepOnePlus System (Applied Biosystems). Briefly, $20 \mu \mathrm{l}$ PCR included $5 \mu \mathrm{l}$ cDNART product, $1 \times$ Master Mix, $0.1 \mathrm{mM}$ microRNA-specific forward primer and $0.1 \mathrm{mM}$ the universal reverse primer. The reactions were incubated at $95^{\circ} \mathrm{C}$ for $10 \mathrm{~min}$, followed by 40 cycles of $95^{\circ} \mathrm{C}$ for $15 \mathrm{~s}$ and $60^{\circ} \mathrm{C}$ for $1 \mathrm{~min}$. All reactions were run in triplicate. The sequence data of the primers are presented in Table 1. RT-qPCR was carried out in compliance with the MIQE guidelines [26] [27].

All qPCR reactions were performed in duplicate, followed by melt curve analysis to verify their specificity and identity. Small nucleolar RNA U6 was selected as the endogenous reference control [28]. Relative microRNA expression levels were calculated using the $\Delta \Delta \mathrm{Ct}$ method as previously described [29].

Peripheral nervous system (PNS) symptoms, gastrointestinal (GI) symptoms, dyshidrosis, angiokeratomas, hearing loss, cornea verticillata and pathological albuminuria were considered typical early symptoms of FD in childhood and adolescence [1] [30] [31]. PNS symptoms were considered the presence of typical crises of neuropathic pain and/or typical acroparesthesias and/or the demonstration of damage of small neurological fibers by Quantitative Sensory Testing (QST) [32] [33]. Dyshidrosis and typical GI symptoms and physical examination were evaluated by questioning [34]. Dermatologist specialist in FD evaluated the presence of angiokeratomas [1]. Hearing loss by alterations of the logoaudiometry test was defined. Presence of cornea verticillata by ophthalmological examination with slit lamp was evaluated [1]. ACR greater than 30 was considered pathological albuminuria [23]. Mayor complications were considered the typical complications of FD adult patients. Cardiac: 1) cardiac fibrosis: presence of typical images in cardiac MRI with gadolinium and/or 2) cardiac ischemia: presence of typical changes in electrocardiogram and/or cardiac perfusion tests and/or 3) cardiac arrhythmia: presence of electrophysiological disorders in electrocardiogram; 4) left ventricular hypertrophy assessed by tissue Doppler echocardiogram and/or cardiac MRI [1] [35]. Renal: decreased eGFR and/or pathological albuminuria. Central nervous system (CNS): cerebral white matter lesions in cerebral MRI angiography and/or clinical stroke were considered by antecedents during the interrogation and physical examination and demonstration of lesion in cerebral MRI angiography [36].

Patients were classified into two groups 1) Patients with urinary excretion profile of microRNAs indicative of renal fibrosis (with increased profibrotic miR and/or decreased miR antifibrotic) and 2) Patients with urinary excretion profile of microRNAs not indicative of renal fibrosis.

\subsection{Statistical Analysis}

Normal distribution of continuous variables was tested using Shapiro-Wilk Test. 
Descriptive statistics are presented as means \pm SD or medians and percentiles. To compare demographic, clinical, and laboratory data, the chi-square or Fisher's exact test, ANOVA, Kruskal-Wallis or the independent $t$ test, were used as appropriate. The Pearson correlation test was used to analyze the association between categorical variables. The Spearman correlation test was used to analyze the association between categorical variables confidence interval was of $95 \%$. Values of $\mathrm{p}<0.05$ were considered of statistical significance. Urinary excretion of microRNAs was studied as a function of age, gender, $\alpha$-gal-A activity, genotype, symptoms, pathological albuminuria and eGFR decrease.

The data were processed in an IBM SPSS Statistics 20 database (Statistical Package for Social Sciences version 21).

\subsection{Statement on Ethics}

The study was carried out in accordance with the Declaration of Helsinki for Human Research and approved by the local Ethics Committee. Written informed consent for inclusion was obtained from each participant.

The patients expressed their willingness to participate in the study and their legal representative or guardian, signed the informed consent; according to local legal regulations.

\section{Results}

Controls: 9 healthy subjects (18.66 \pm 13.43 years) were studied, 4 males and 5 females. All presented normal eGFR without pathological albuminuria.

Screening period: a total of 73 FD patients were evaluated during the study period, 12 patients had inclusion criteria.

FD population: 12 patients $(10.33 \pm 3.93$ years $)$ were studied, 5 males and 7 females. Patients presented two different genotypes: L415P (6 patients) and E398X (6 patients). 5/5 males (100\%) and 1/7 females (14.28\%) had decreased $\alpha$-gal-A activity. The mean value of eGFR was $152.33 \pm 48.39 \mathrm{ml} / \mathrm{min} / 1.73 \mathrm{~m}^{2}$ and ACR was $21.75 \pm 37.13 \mathrm{mg} / \mathrm{g} .1 / 5$ males $(20.0 \%)$ and $1 / 7$ (14.28\%) females had microalbuminuria. No patient had proteinuria.

There were no significant differences between the age of both populations studied $(\mathrm{p}=0.054)$.

Frequency of clinical manifestations in FD patients is shown in Table 2.

The comparison of the urinary excretion of microRNAs families studied, between control group and FD patients are shown in Figure 1.

The urinary excretion profile of microRNAs indicative of renal fibrosis was present in 4 patients (2 with L415P genotype and 2 with E398X genotype), all for the decrease of miR-29 and/or miR-200. No patient presented increased miR-21, miR-192 and/or miR-433.

Table 3 shows the correlation between the profile of urinary excretion of microRNAs indicative of renal fibrosis and the clinical characteristics of FD patients. 
Table 2. Frequency of clinical manifestations in Fabry disease patients.

\begin{tabular}{cc}
\hline & Frequency (\%) \\
\hline Early symptoms & $3 / 12(25)$ \\
PNS symptoms & $3 / 12(25)$ \\
GI symptoms & $3 / 12(25)$ \\
Dyshidrosis & $3 / 12(25)$ \\
Angiokeratomas & $0 / 12(0)$ \\
Hearingloss & $1 / 12(8.33)$ \\
Cornea verticillata & $2 / 12(16.66)$ \\
Pathological albuminuria & \\
Mayor complications & $0 / 12(0)$ \\
Cardiac & $0 / 12(0)$ \\
Renal & $0 / 12(0)$ \\
CNS &
\end{tabular}

PNS: peripheral nervous system; GI: gastrointestinal; CNS: central nervous system.

Table 3. Correlation between urinary excretion of microRNAs indicative of renal fibrosis and clinical characteristics of Fabry disease patients.

\begin{tabular}{cc}
\hline Variable & $\begin{array}{c}\text { Urinary profile excretion of microRNAs indicative of renal } \\
\text { fibrosis. Pearson correlation (p value) }\end{array}$ \\
\hline Age & $0.031(0.923)$ \\
Gender & $0.478(0.116)$ \\
$\boldsymbol{\alpha}$-gal-A activity & $0.707(<0.01)^{*}$ \\
Genotype & $0.000(1.000)$ \\
Symptoms & $0.250(0.433)$ \\
Pathological albuminuria & $0.425(0.169)$ \\
eGFRdecrease & $0.166(0.607)$ \\
\hline
\end{tabular}

${ }^{*}$ Correlation is significant at the 0.01 level. eGFR: estimated glomerular filtration rate.

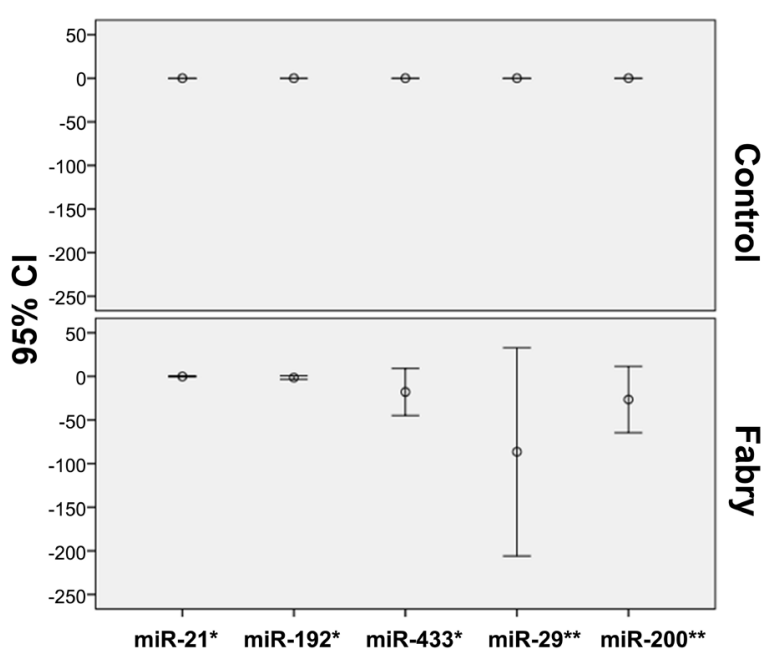

Figure 1. Comparison of urinary microRNAs excretion between control group and Fabry disease patients of pro-fibrotic microRNAs ${ }^{\star}$ and anti-fibrotic microRNAs ${ }^{\star *}$. 


\section{Discussion}

Most male patients and a significant number of female patients will eventually develop nephropathy without treatment [37] [38]. Nephropathy is one of the major complications of FD and mainly includes reduced glomerular filtration rate and proteinuria [38].

One of the challenges in the field of research of FD nephropathy is the search for noninvasive biomarkers of kidney damage in pre-albuminuric stages, since those currently available and used routinely, lack sensitivity to detect early damage [39].

In our study, we analyzed the urinary excretion of microRNAs in a population of young FD patients and mild or absent nephropathy. Because there are no precedents in the subject, the five families of microRNAs recognized were selected, related to the process of renal fibrosis via TGF- $\beta$ in other nephropathies, such as diabetic nephropathy [40] [41].

During fibrosis, TGF- $\beta$ is capable of inducing many fibrogenic genes, such as extracelular matrix proteins, via $\operatorname{Smad} 2, \operatorname{Smad} 3$ or mitogen-activated protein kinases (MAPKs) [14] [40] [41]. TGF- $\beta 1$ induces miR-21, miR-192 and miR-433, but suppresses the miR-29 and miR-200 families [14] [40]. Recent evidence demonstrates that TGF- $\beta$ signaling induces the synthesis of fibrosis-related microRNAs either by increasing transcription, or by enhancing post-transcriptional processing of primary microRNA transcript [40]. There are multiple steps of the biogenesis of microRNAs [41]; its detailed description is beyond the scope of this paper. The mature microRNA induces the RISC complex to bind to the $3^{\prime}$ untranslated region of a target messenger RNA (mRNA). This will result in post-transcriptional gene silencing by mRNA degradation or by translation inhibition. Therefore, microRNAs is able to suppress target gene expression by mRNA degradation, translation inhibition or transcriptional inhibition [42].

In this study, a population of patients with two classical mutations of the GLA gene was compared with healthy subjects with similar demographic characteristics. Despite being patients with mutations that originate the severe FD phenotype, because they are young patients, a low prevalence of both early and late complications of FD was observed.

Because FD is a disease of inheritance $\mathrm{X}$-linked, in patients with classical mutations of the GLA gene which cause severe deficits of $\alpha$-gal-A activity, affected men develop symptoms at an early age and in women that happens at a variable age and severity.

It was observed that the profile of urinary excretion of microRNAs indicative of renal fibrosis present in the patients was produced by decrease of the anti-fibrotic microRNAs (miR-29; miR-200) and not by increase of the pro-fibrotic microRNAs (miR-21; miR-192; miR-433).

In our results, the only variable associated with statistical significance for a profile of urinary excretion of microRNAs indicative of renal fibrosis was low $\alpha$-gal-A activity. No statistically significant association was found with the rest of the variables studied, even with respect to the sex of the patients. 
Although the small size of the population studied may represent a statistical limitation, other studies devoted to similar purposes were carried out with an analogous number of patients. Therefore, considering the low frequency of FD, this work could contribute to the knowledge of the subject.

Young FD patients with classical mutations of the GLA gene and mild or absent nephropathy presented a profile of urinary excretion of microRNAs indicative of renal fibrosis, even in the pre-albumin stage. This profile was produced by decreasing of microRNAs that are down regulated by TGF- $\beta$ and was significantly correlated with the decrease in $\alpha$-gal-A activity independently of the other variables studied. Our results would support the theory of initiating ERT treatment in FD patients with decreased levels of $\alpha$-gal-A in asymptomatic patients with classical GLA mutations.

The decrease of anti-fibrotic microRNAs and the non-increase of pro-fibrotic microRNAs could suggest a regulation of microRNAs not mediated by TGF- $\beta 1$ in FD nephropathy.

\section{Conclusion}

Our results show that young FD patients with classical mutations of the GLA gene and mild or absent nephropathy could present a profile of urinary excretion of microRNAs indicative of renal fibrosis associated with statistical significance at decreased $\alpha$-gal-A activity independently of the other variables studied. A urinary excretion profile of microRNAs could be used as non-invasive biomarkers of early kidney damage due to $\mathrm{FD}$, even in pre-albuminuric stages. The decrease of anti-fibrotic microRNAs and the non-increase of pro-fibrotic microRNAs could suggest a regulation of microRNAs not mediated by TGF- $\beta$ in FD nephropathy. Longitudinal studies with a larger number of patients would be necessary to confirm this finding.

\section{Data Availability Statement}

The data used to support the findings of this study are restricted by the Ethic Committee of the Instituto Universitario Italiano de Rosario in order to protect patients privacity. Data are available from for researchers who meet the criteria for access to confidential data.

\section{Conflicts of Interest}

SJ declarations to have received financial contributions from the companies SANOFI-Genzyme, Shire HGT and Biomarin for dissertations on Fabry disease and Mucopolisacaridosis and financial contributions from SANOFI-GENZYME for research projects; the other authors do not have conflicts of interest related to the contents of this work.

\section{Funding Statement}

The present work was carried out with the financial support of the SANOFI- 
Genzyme company of Argentina, for the purchase of medical supplies used in the laboratory.

\section{References}

[1] Germain, D.P. (2010) Fabry Disease. Orphanet Journal of Rare Diseases, 5, 30. https://doi.org/10.1186/1750-1172-5-30

[2] Tsutsumi, O., Sato, M., Sato, K., Mizuno, M. and Sakamoto, S. (1985) Early Prenatal Diagnosis of Inborn Error of Metabolism: A Case Report of a Fetus Affected with Fabry's Disease. Asia-Oceania Journal of Obstetrics and Gynaecology, 11, 39-45. https://doi.org/10.1111/j.1447-0756.1985.tb00045.x

[3] Hopkin, R.J., Bissler, J., Banikazemi, M., Clarke, L., Eng, C.M., Germain, D.P., Lemay, R., Tylki-Szymanska, A. and Wilcox, W.R. (2008) Characterization of Fabry Disease in 352 Pediatric Patients in the Fabry Registry. Pediatric Research, 64, 550-555. https://doi.org/10.1203/PDR.0b013e318183f132

[4] Waldek, S., Patel, M.R., Banikazemi, M., Lemay, R. and Lee, P. (2009) Life Expectancy and Cause of Death in Males and Females with Fabry Disease: Findings from the Fabry Registry. Genetics in Medicine, 11, 790-796. https://doi.org/10.1097/GIM.0b013e3181bb05bb

[5] Najafian, B., Mauer, M., Hopkin, R.J. and Svarstad, E. (2013) Renal Complications of Fabry Disease in Children. Pediatric Nephrology, 28, 679-687. https://doi.org/10.1007/s00467-012-2222-9

[6] Sheth, K.J., Roth, D.A. and Adams, M.B. (1983) Early Renal Failure in Fabry's Disease. American Journal of Kidney Diseases, 2, 651-654. https://doi.org/10.1016/S0272-6386(83)80047-X

[7] Tondel, C., Bostad, L., Hirth, A. and Svarstad, E. (2008) Renal Biopsy Findings in Children and Adolescents with Fabry Disease and Minimal Albuminuria. American Journal of Kidney Diseases, 51, 767-776. https://doi.org/10.1053/j.ajkd.2007.12.032

[8] Fogo, A.B., Bostad, L., Svarstad, E., Cook, W.J., Moll, S., Barbey, F., Geldenhuys, L., West, M., Ferluga, D., Vujkovac, B., Howie, A.J., Burns, A., Reeve, R., Waldek, S., Noel, L.H., Grunfeld, J.P., Valbuena, C., Oliveira, J.P., Muller, J., Breunig, F., Zhang, X., Warnock, D.G. and All Members of the International Study Group of Fabry (2010) Scoring System for Renal Pathology in Fabry Disease: Report of the International Study Group of Fabry Nephropathy (ISGFN). Nephrology Dialysis Transplantation, 25, 2168-2177. https://doi.org/10.1093/ndt/gfp528

[9] Perretta, F., Antongiovanni, N. and Jaurretche, S. (2017) Early Renal Involvement in a Girl with Classic Fabry Disease. Case Reports in Nephrology, 2017, Article ID: 9543079. https://doi.org/10.1155/2017/9543079

[10] Trimarchi, H., Canzonieri, R., Schiel, A., Costales-Collaguazo, C., Politei, J., Stern, A., Paulero, M., Rengel, T., Andrews, J., Forrester, M., Lombi, M., Pomeranz, V., Iriarte, R., Muryan, A., Zotta, E., Sanchez-Nino, M.D. and Ortiz, A. (2016) Increased Urinary CD80 Excretion and Podocyturia in Fabry Disease. Journal of Translational Medicine, 14, 289.

[11] Trimarchi, H., Canzonieri, R., Schiel, A., Politei, J., Stern, A., Andrews, J., Paulero, M., Rengel, T., Araoz, A., Forrester, M., Lombi, F., Pomeranz, V., Iriarte, R., Young, P., Muryan, A. and Zotta, E. (2016) Podocyturia Is Significantly Elevated in Untreated vs Treated Fabryadult Patients. Journal of Nephrology, 29, 791-797. https://doi.org/10.1007/s40620-016-0271-z

[12] Aguiar, P., Azevedo, O., Pinto, R., Marino, J., Baker, R., Cardoso, C., Ducla Soares, 
J.L. and Hughes, D. (2017) New Biomarkers Defining a Novel Early stage of Fabry Nephropathy: A Diagnostic Test Study. Molecular Genetics and Metabolism, 121, 162-169. https://doi.org/10.1016/j.ymgme.2017.05.007

[13] Trionfini, P., Benigni, A. and Remuzzi, G. (2015) MicroRNAs in Kidney Physiology and Disease. Nature Reviews Nephrology, 11, 23-33. https://doi.org/10.1038/nrneph.2014.202

[14] Chung, A.C. and Lan, H.Y. (2015) MicroRNAs in Renal Fibrosis. Frontiers in Physiology, 6, 50. https://doi.org/10.3389/fphys.2015.00050

[15] Aguiar, P., Costa, M.C., Azevedo, O., Fiúza, M., Marino, J., Soares, J.L.D. and Enguita, F.J. (2017) MicroRNAs in Fabry Disease: Role as Biomarkers and a Possible Pathophysiological Pathway. Molecular Genetics and Metabolism, 120, S17.

[16] Aguiar, P., Azevedo, O., Pinto, R., Marino, J., Cardoso, C., Soares, J.L.D. and Hughes, D. (2017) Increase Synthesis of Collagen Type I: An Early Event in Fabry Cardiomyopathy. New Biomarkers? Molecular Genetics and Metabolism, 120, S17-S18.

[17] Pasqualim, G.P.É., Angrezani, J., Giugliani, R., Vairo, F. and Matte, U. (2017) MicroRNA and Gene Expression Studies Provide Novel Information to Understand Heart Disease in Women with Fabry Disease. Molecular Genetics and Metabolism, 120, S106.

[18] Cammarata, G., Scalia, S., Colomba, P., Zizzo, C., Pisani, A., Riccio, E., Montalbano, M., Alessandro, R., Giordano, A. and Duro, G. (2018) A Pilot Study of Circulating MicroRNAs as Potential Biomarkers of Fabry Disease. Oncotarget, 9, 27333-27345. https://doi.org/10.18632/oncotarget.25542

[19] Hopkin, R.J., Jefferies, J.L., Laney, D.A., Lawson, V.H., Mauer, M., Taylor, M.R., Wilcox, W.R. and Fabry, P. (2016) The Management and Treatment of Children with Fabry Disease: A United States-Based Perspective. Molecular Genetics and Metabolism, 117, 104-113. https://doi.org/10.1016/j.ymgme.2015.10.007

[20] Eng, C.M., Resnick-Silverman, L.A., Niehaus, D.J., Astrin, K.H. and Desnick, R.J. (1993) Nature and Frequency of Mutations in the Alpha-Galactosidase A Gene That Cause Fabry Disease. The American Journal of Human Genetics, 53, 1186-1197.

[21] Schirinzi, A., Centra, M., Prattichizzo, C., Gigante, M., De Fabritiis, M., Giancaspro, V., Petrarulo, F., Santacroce, R., Margaglione, M., Gesualdo, L. and Ranieri, E. (2008) Identification of GLA Gene Deletions in Fabry Patients by Multiplex Ligation-Dependent Probe Amplification (MLPA). Molecular Genetics and Metabolism, 94, 382-385. https://doi.org/10.1016/j.ymgme.2008.03.017

[22] Li, Y., Scott, C.R., Chamoles, N.A., Ghavami, A., Pinto, B.M., Turecek, F. and Gelb, M.H. (2004) Direct Multiplex Assay of Lysosomal Enzymes in Dried Blood Spots for Newborn Screening. Clinical Chemistry, 50, 1785-1796.

https://doi.org/10.1373/clinchem.2004.035907

[23] Peralta, C.A., Shlipak, M.G., Judd, S., Cushman, M., McClellan, W., Zakai, N.A., Safford, M.M., Zhang, X., Muntner, P. and Warnock, D. (2011) Detection of Chronic Kidney Disease with Creatinine, Cystatin C, and Urine Albumin-to-Creatinine Ratio and Association with Progression to End-Stage Renal Disease and Mortality. JAMA, 305, 1545-1552. https://doi.org/10.1001/jama.2011.468

[24] Tondel, C., Ramaswami, U., Aakre, K.M., Wijburg, F., Bouwman, M. and Svarstad, E. (2010) Monitoring Renal Function in Children with Fabry Disease: Comparisons of Measured and Creatinine-Based Estimated Glomerular Filtration Rate. Nephrology Dialysis Transplantation, 25, 1507-1513. https://doi.org/10.1093/ndt/gfp658

[25] Chen, C., Ridzon, D.A., Broomer, A.J., Zhou, Z., Lee, D.H., Nguyen, J.T., Barbisin, 
M., Xu, N.L., Mahuvakar, V.R., Andersen, M.R., Lao, K.Q., Livak, K.J. and Guegler, K.J. (2005) Real-Time Quantification of microRNAs by Stem-Loop RT-PCR. Nucleic Acids Research, 33, e179.

[26] Bustin, S.A., Beaulieu, J.F., Huggett, J., Jaggi, R., Kibenge, F.S., Olsvik, P.A., Penning, L.C. and Toegel, S. (2010) MIQE Precis: Practical Implementation of Minimum Standard Guidelines for Fluorescence-Based Quantitative Real-Time PCR Experiments. BMC Molecular Biology, 11, 74. https://doi.org/10.1186/1471-2199-11-74

[27] Bustin, S.A., Benes, V., Garson, J.A., Hellemans, J., Huggett, J., Kubista, M., Mueller, R., Nolan, T., Pfaffl, M.W., Shipley, G.L., Vandesompele, J. and Wittwer, C.T. (2009) The MIQE Guidelines: Minimum Information for Publication of Quantitative Real-Time PCR Experiments. Clinical Chemistry, 55, 611-622. https://doi.org/10.1373/clinchem.2008.112797

[28] Mestdagh, P., Van Vlierberghe, P., De Weer, A., Muth, D., Westermann, F., Speleman, F. and Vandesompele, J. (2009) A Novel and Universal Method for microRNA RT-qPCR Data Normalization. Genome Biology, 10, R64.

[29] Livak, K.J. and Schmittgen, T.D. (2001) Analysis of Relative Gene Expression Data Using Real-Time Quantitative PCR and the 2(-Delta DeltaC(T)) Method. Methods, 25, 402-408. https://doi.org/10.1006/meth.2001.1262

[30] Ramaswami, U., Whybra, C., Parini, R., Pintos-Morell, G., Mehta, A., Sunder-Plassmann, G., Widmer, U., Beck, M. and F.O.S.E. Investigators (2006) Clinical Manifestations of Fabry Disease in Children: Data from the Fabry Outcome Survey. Acta Paediatrica, 95, 86-92. https://doi.org/10.1080/08035250500275022

[31] Laney, D.A., Peck, D.S., Atherton, A.M., Manwaring, L.P., Christensen, K.M., Shankar, S.P., Grange, D.K., Wilcox, W.R. and Hopkin, R.J. (2015) Fabry Disease in Infancy and Early Childhood: A Systematic Literature Review. Genetics in Medicine, 17, 323-330. https://doi.org/10.1038/gim.2014.120

[32] Liguori, R., Di Stasi, V., Bugiardini, E., Mignani, R., Burlina, A., Borsini, W., Baruzzi, A., Montagna, P. and Donadio, V. (2010) Small Fiber Neuropathy in Female Patients with Fabry Disease. Muscle \& Nerve, 41, 409-412. https://doi.org/10.1002/mus.21606

[33] Biegstraaten, M., Hollak, C.E., Bakkers, M., Faber, C.G., Aerts, J.M. and van Schaik, I.N. (2012) Small Fiber Neuropathy in Fabry Disease. Molecular Genetics and Metabolism, 106, 135-141. https://doi.org/10.1016/j.ymgme.2012.03.010

[34] Zar-Kessler, C., Karaa, A., Sims, K.B., Clarke, V. and Kuo, B. (2016) Understanding the Gastrointestinal Manifestations of Fabry Disease: Promoting Prompt Diagnosis. Therapeutic Advances in Gastroenterology, 9, 626-634. https://doi.org/10.1177/1756283X16642936

[35] Seydelmann, N., Wanner, C., Stork, S., Ertl, G. and Weidemann, F. (2015) Fabry Disease and the Heart. Best Practice \& Research: Clinical Endocrinology \& Metabolism, 29, 195-204. https://doi.org/10.1016/j.beem.2014.10.003

[36] Tuttolomondo, A., Pecoraro, R., Simonetta, I., Miceli, S., Arnao, V., Licata, G. and Pinto, A. (2013) Neurological Complications of Anderson-Fabry Disease. Current Pharmaceutical Design, 19, 6014-6030. https://doi.org/10.2174/13816128113199990387

[37] Schiffmann, R., Warnock, D.G., Banikazemi, M., Bultas, J., Linthorst, G.E., Packman, S., Sorensen, S.A., Wilcox, W.R. and Desnick, R.J. (2009) Fabry Disease: Progression of Nephropathy, and Prevalence of Cardiac and Cerebrovascular Events before Enzyme Replacement Therapy. Nephrology Dialysis Transplantation, 24, 
2102-2111. https://doi.org/10.1093/ndt/gfp031

[38] Jaurretche, S., Antogiovanni, N. and Perreta, F. (2017) Prevalence of Chronic Kidney Disease in Fabry Disease Patients: Multicenter Cross Sectional Study in Argentina. Molecular Genetics and Metabolism, 12, 41-43.

https://doi.org/10.1016/j.ymgmr.2017.05.007

[39] Schiffmann, R., Waldek, S., Benigni, A. and Auray-Blais, C. (2010) Biomarkers of Fabry Disease Nephropathy. Clinical Journal of the American Society of Nephrolo$g y$, 5, 360-364. https://doi.org/10.2215/CJN.06090809

[40] Meng, X.M., Tang, P.M., Li, J. and Lan, H.Y. (2015) TGF-Beta/Smad Signaling in Renal Fibrosis. Frontiers in Physiology, 6, 82.

https://doi.org/10.3389/fphys.2015.00082

[41] Bottinger, E.P. (2007) TGF-Beta in Renal Injury and Disease. Seminars in Nephrology, 27, 309-320. https://doi.org/10.1016/j.semnephrol.2007.02.009

[42] Bartel, D.P. (2018) Metazoan MicroRNAs. Cell, 173, 20-51.

https://doi.org/10.1016/j.cell.2018.03.006 